\title{
Evaluación de la Escala de Prosocialidad de Caprara, Steca, Zelli y Capanna en Estudiantes Universitarios Chilenos
}

\author{
Evaluation of the Prosociality Scale of Caprara, Steca, Zelli and Capanna in Chilean \\ Undergraduat Students
}

\author{
Manuel Mieres-Chacaltana ${ }^{1}$, Sonia Salvo-Garrido ${ }^{2}$ y Marianela Denegri-Coria ${ }^{3}$
}

\section{Resumen}

El objetivo de este estudio fue la evaluación de la Escala de Prosocialidad Adulta de Caprara, Steca, Zelli y Capanna (2005) en estudiantes universitarios chilenos de carreras de pedagogía ( $\mathrm{N}=860 ; M=20.72$ años; $D E=2.5$ ). El proceso comprendió dos fases. Primero, la traducción de la escala al español siguiendo los criterios de traducción, re traducción y equivalencia de contenido con el original. Luego, la evaluación de su consistencia interna, fiabilidad, estructura factorial y validez convergente, mediante un análisis exploratorio de ecuaciones estructurales (ESEM). Los resultados arrojaron un buen nivel unidimensional de la escala (factor general, $\mathrm{ECV}=.749 ; \omega \mathrm{h}=.858$ ) un adecuado nivel de fiabilidad ( $\omega$ del factor general=.905) y se ratificó su adecuación al contexto investigado con un buen nivel de ajuste (RMSEA=.035; CFI=.993; TLI=.982).

Palabras clave: prosocialidad, propiedades psicométricas, evaluación

\begin{abstract}
This study's purpose was to evaluate the Adult Prosociality Scale of Caprara, Steca, Zelli, and Capanna (2005) applied to Chilean university students registered in programs leading to obtaining a bachelor's Degree in Education ( $\mathrm{N}=860 ; \mathrm{M}=20.72$ years; $\mathrm{SD}=2.5)$. The process involved two phases. The first included turning the scale into Spanish according to the criteria of translation, re-translation, and equivalence of content with the original. The second one involved evaluating its internal consistency, reliability, factor structure, and convergent validity through an exploratory analysis of structural equations (ESEM). The results showed an outstanding one-dimensional level of the scale (general factor, $\mathrm{ECV}=.76, \omega \mathrm{h}=.864$ ), an adequate level of reliability (general factor $\omega=.904$ ) and ratified its adequacy to the subject under study with a proper level of adjustment (RMSEA=.034; CFI=.993; TLI=.983).
\end{abstract}

Keywords: prosociality, psychometric properties, evaluation

Agradecimientos: Trabajo financiado parcialmente por el proyecto FONDECYT 1160429

\footnotetext{
${ }^{1}$ Magíster en Ciencias Sociales Aplicadas y Doctorando en Ciencias Sociales, Universidad de La Frontera, Académico de la Universidad Católica de Temuco, Calle Rudecindo Ortega 02950, Chile. Código postal: 4780071. Tel.: +56-45-2553803. Correo: mieres@ uct.cl

${ }^{2}$ Doctora en Estadística Multivariante, Académica Departamento de Matemática y Estadística. Investigadora del Laboratorio de Investigación en Ciencias Sociales Aplicadas (LICSA) de la Universidad de La Frontera, Chile. Código postal: 4811230. Tel.: +56-0452325670. Correo: sonia.salvo@ufrontera.cl (correspondencia).

${ }^{3}$ Dra. en Psicología, Departamento de Psicología. Directora del Núcleo de Ciencias Sociales y Humanidades de la Universidad de La Frontera, Chile. Código postal: 4811230. Tel.: +56-45-2325670. Correo: marianela.denegri@ufrontera.cl

Revista Iberoamericana de Diagnóstico y Evaluación - e Avaliação Psicológica. RIDEP · №56 · Vol.3 · 21-32 · 2020

ISSN: 1135-3848 print /2183-6051online
} 


\section{Introducción}

El estudio de la prosocialidad presenta un renovado interés en las últimas dos décadas. Las investigaciones han confirmado sistemáticamente su relevancia, centrada en los beneficios que tiene para cada persona y para la sociedad en general (Caprara, Alessandri, \& Eisenberg, 2012). Por ejemplo, en relación con el mayor compromiso cívico (Luengo et al., 2014), el mejoramiento del vínculo social y el aplacamiento de la agresividad (Carlo, Mestre, Samper, Tur, \& Armenta, 2010).

La prosocialidad se define como un comportamiento voluntario destinado a beneficiar a otro (Eisenberg, Spinrad, \& Knafo-Noam, 2015), con algunos disensos entre las formas de abordar sus componentes y, especialmente, los motivos que subyacen a ella. En esta línea, algunos autores asumen prosocialidad y altruismo como expresiones equivalentes (Roche, 1982; Warneken \& Tomasello, 2009). Ello implica considerar que detrás de una acción prosocial no existe una anticipación de ningún tipo de recompensa. En cambio, otros afirman que el comportamiento prosocial puede explicarse desde una amplia gama de motivos, incluido el egoísmo (Carlo \& Randall, 2002; Eisenberg et al., 2015).

Con relación a la investigación de la prosocialidad en el ámbito educativo, algunos estudios han explorado la relación entre ésta con el aumento del aprendizaje y/o el mejoramiento de la conducta en estudiantes preadolescentes y adolescentes (Caprara et al., 2014; Caprara, Luengo, Zuffiano, Gerbino, \& Pastorelli, 2015; Inglés, Martínez-González, Valle, GarcíaFernández, \& Ruiz-Esteban, 2011). Otros estudios enfatizan los rasgos prosociales de los maestros como capacidades indispensables para el logro de distintos objetivos formativos. De acuerdo con Riley (2010), la forma en que los maestros forman y mantienen las relaciones en el salón de clases y el personal es crucial para el éxito de su trabajo. Ello incluye la capacidad de ponerse en el lugar de los estudiantes y desplegar acciones de ayuda acordes con las necesidades manifestadas por éstos. Igualmente, el apoyo emocional del docente, y su capacidad empática, mejora las relaciones entre compañeros en el aula (Cappella, Kim, Neal, \& Jackson, 2013; Denisenkova \& Nisskaya, 2016; Shin \& Ryan, 2017). Asimismo, contribuyen al ajuste emocional del estudiante (Sointu, Savolainen, Lappalainen, \& Lambert, 2017). Del mismo modo, conductas prosociales entre maestros, como compartir información y otros recursos, facilita la gestión del aula, previene y disminuye el comportamiento problemático (Ertesvåg, 2014). Otros hallazgos han reportado relaciones bidireccionales positivas, a lo largo del tiempo, entre clima escolar y conducta prosocial en adolescentes (Luengo et al., 2017).

En el caso específico de Chile, si bien la legislación educacional actual no incorpora explícitamente el concepto de prosocialidad, sí promueve el fomento de la paz, la convivencia social y la democracia, de acuerdo con el Ministerio de Educación (MINEDUC, 2010). Cabe entender esto en el marco del devenir de las políticas educativas propiciadas desde los años noventa con el retorno a la democracia y que tiene como antecedente relevante el informe sobre la educación para el siglo XXI, de la Organización de las Naciones Unidas para la Educación, la Ciencia y la Cultura (UNESCO, 1996). Uno de los pilares de la educación del futuro, según dicho informe, lo constituye el "aprender a vivir juntos" (UNESCO, 1996, p.103). Para ello, la escuela debe, desde sus niveles más tempranos, otorgar oportunidades para el trabajo cooperativo y la participación en actividades sociales solidarias y de ayuda (UNESCO, 1996, p.105-106). Actualmente, y en consonancia con lo anterior, la docencia debe asumir una perspectiva integral y propiciar un "aprendizaje social, cultural, intelectual y ético" (MINEDUC, 2015, p.23). Ello configura un vínculo dialéctico "entre los procesos individuales y sociales, por tanto, como ámbito de formación y responsabilidad de los docentes" (MINEDUC, 2015, p.23). En consecuencia, esto representa un desafío, tanto para los profesores en ejercicio, como para las instituciones a cargo de la formación inicial docente. En esta última área, constituye, además, una oportunidad de fortalecer la educación social y emocional de los maestros. Esto, ante la creciente evidencia de que la educación social y emocional está relacionada con actitudes académicas positivas y un mayor rendimiento académico, con un aumento del comportamiento prosocial y con una disminución del comportamiento antisocial, la ansiedad, la 
depresión y el suicidio (Cefai, Bartolo, Cavioni, \& Downes, 2018).

En esta misma línea, varios autores coinciden acerca de la necesidad de estudiar la conducta prosocial en la adultez en ámbitos profesionales específicos, como el de la educación. Ello, dado que la prosocialidad varía con la edad (Contreras \& Reyes, 2009) y, entre otros factores, es dependiente de la situación (González, 2000). En el caso de la formación inicial docente, la edad constituye un factor a considerar, si se tiene en cuenta que muchas personas inician sus estudios de pedagogía entre los 17 y 19 años. Estas edades están comprendidas dentro de lo que se denomina adolescencia tardía, que, de acuerdo a lo planteado por Fondo de las Naciones Unidas para la Infancia (UNICEF, 2011) va desde los 15 hasta los 19 años. Estadísticas recientes indican que en 2016 casi un $18 \%$ de la matrícula universitaria en Chile en 2016 correspondía a estudiantes entre 17 y 19 años (Ministerio de Educación de Chile, 2017). En términos teóricos, se aprecia un solapamiento de edades con la primera fase de la adultez, más precisamente, con la adultez emergente, que se desarrolla entre los 18 y 29 años. Fase transicional a la vida adulta, caracterizada por la exploración, la construcción de identidad y coincidente con el paso por la universidad de los jóvenes chilenos (Barrera \& Vinet, 2017). Si se toman los datos anteriores, aplicados a este rango de edad, entonces, la matrícula universitaria incluye casi el 86\% (Ministerio de Educación de Chile, 2017).

Por ello, si se espera estudiar la prosocialidad en la edad adulta, ello incluye la necesidad de construcción de instrumentos de medición adecuados a este segmento etario (Auné, Blum, Abal, Lozzia, \& Attorresi, 2014). En este ámbito Caprara et al. (2005) desarrollaron en Italia una escala para evaluar la prosocialidad en adultos. Sus 16 ítems reflejan comportamientos y sentimientos, asociados con cuatro tipos de acciones: compartir, cuidar, ayudar y sentir empatía. Al incluir la empatía - situación discutible para otros estudios -, Caprara et al. (2005, p.80) fundamentan que en la edad adulta, los motivos o predisposiciones empáticas no son meramente un correlato de la tendencia a actuar prosocialmente, sino más bien, son parte integral de tal tendencia. Incluso, los rasgos empáticos figuran también como dimensión de la prosocialidad en otros instrumentos diseñados para medir este constructo en niños y adolescentes (Auné \& Attorresi, 2017; Martorell, González, Aloy, \& Ferris, 1995; Martorell, González, Ordóñez, \& Gómez, 2011), adultos (Auné, Abal, \& Attorresi, 2016). O bien, como reactivos asociados con otras dimensiones de la prosocialidad, como la de confortar, aplicado con población adulta (Auné \& Attorresi, 2017). Ahora bien, reconocer e, inclusive, operacionalizar diversas dimensiones teóricas de la prosocialidad no garantiza que estas emerjan cuando se aplican los instrumentos de medición. En otras palabras, aun definiendo dimensiones previas en un instrumento, los resultados podrían mostrar unidimensionalidad si hubiera alta correlación entre todos los ítems (Balabanian \& Lemos, 2018).

Aparte de la investigación original, el instrumento ha sido utilizado y evaluado en otros estudios con adolescentes y adultos de nacionalidad italiana, española, colombiana y argentina (Biagioli, Prandi, Giuliani, Nyatanga, \& Fida, 2016; Caprara et al., 2012; Caprara \& Steca, 2007; Capurso, Dennis, \& Piobbico, 2016; Luengo et al., 2017; Rodríguez, Mesurado, Oñate, Guerra, \& Menghi, 2017; Rodríguez \& Moreno, 2016; Suriá, 2017). En el caso de las pruebas realizadas en países latinoamericanos, la escala original tuvo modificaciones, con el fin de adaptarla mejor a las realidades culturales. La evaluación hecha por Rodríguez et al. (2017) con una muestra de adolescentes argentinos definió un modelo con 10 de los 16 ítems originales y dos dimensiones: conducta prosocial y empatía y apoyo emocional. En Colombia, Luengo et al. (2017), lo aplicaron a una muestra de escolares adolescentes y sus padres, utilizando, respectivamente, cuatro y tres ítems.

Sobre la base de las consideraciones expuestas, el objetivo de este trabajo fue evaluar la fiabilidad, estructura factorial y validez convergente de la Escala de Prosocialidad Adulta de Caprara et al. (2005) en estudiantes chilenos de pedagogía. El propósito es desarrollar una fase de estudios previos que permita en el futuro contar con instrumentos para medir la prosocialidad en términos generales y personales. Con ello, generar conocimiento que sirva de insumo para orientar los procesos de educación social y emocional de los futuros profesores. 


\section{Método}

\section{Participantes}

La población definida para este estudio quedó constituida por los estudiantes de pedagogía de tres universidades (dos privadas y una pública) situadas en la Región de la Araucanía, Chile. El total aproximado ascendió a 3,995 estudiantes.

La selección del número de participantes se realizó mediante un muestreo probabilístico estratificado, con una confiabilidad del $95 \%$ y un $2 \%$ de error de muestreo. Se supuso máxima variabilidad, es decir, $\mathrm{p}=\mathrm{q}=0,5$ (Scheaffer, Mendenhall, \& Ott, 1987). La muestra del estudio se conformó con 860 estudiantes $(43.4 \%$ hombres y $56.6 \%$ mujeres). La edad media de los participantes se situó en 20.72 años, con una desviación estándar de 2.5 años. La Tabla 1 detalla los datos referidos al muestreo.

\section{Instrumento}

Se usó la escala para medir prosocialidad en adultos, construida por Caprara et al. (2005). El instrumento se tradujo al español adaptando sus reactivos a la población chilena, como se muestra en la Tabla 2. Esta escala contiene 16 ítems, cada uno asociado a cinco categorías en una escala ordinal. La traducción de sus etiquetas fue: $1=$ Nunca, $2=$ Ocasionalmente, $3=\mathrm{A}$ veces, $4=\mathrm{A}$ menudo, y 5=Siempre. Cada uno de los ítems se asocia con, al menos, una de las dimensiones teóricas que, según Caprara el al. (2005), componen el comportamiento prosocial: ayudar, compartir, cuidar y empatizar.

\section{Procedimiento}

En primer lugar, se procedió a la traducción y adaptación idiomática del instrumento original. Para ello se realizó la traducción al español por dos traductores independientes (Trust, 1997). Luego, se hizo la re-traducción por un nuevo traductor bilingüe (Maneersriwongul \& Dixon, 2004). Una vez realizado esto, se sometió la traducción al juicio de dos jueces expertos para la validez de contenido (Escobar \& Cuervo, 2008) para asegurar la equivalencia de contenido, semántica, técnica, de criterio y conceptual con el original. Se Calculó el índice de validez de contenido (Lawshe, 1975), el cual dio un valor de
1, indicando que el conjunto de ítems es adecuado (Davis, 1992). Asimismo, se comparó dicha traducción con otra utilizada en Argentina (Rodríguez et al., 2017), país que presenta un contexto lingüístico similar. Se constató similitud y equivalencia entre ambas traducciones.

Una vez traducida y normalizada la escala, se definió la muestra y se contactó a los estudiantes. En algunos casos, de manera directa; en otros, indirectamente, a través de los directores de los respectivos programas. La participación fue voluntaria y anónima. A los estudiantes, se les solicitó firmar una carta de consentimiento informado. En ésta, se explicaban los objetivos y alcances del estudio y se garantizaba la confidencialidad de los datos recogidos. Cabe agregar que la anonimia, junto con resguardar la identidad de cada participante, influye en la disminución de la deseabilidad social (Fisher, 1993).

\section{Análisis de datos}

Se estimó un modelo de ecuaciones estructurales bifactor, constituido por un factor general de prosocialidad y cuatro factores específicos concernientes a las dimensiones teóricas propuestas por Caprara et al. (2005). Los modelos bifactor (Howard, Gagné, Morin, \& Forest, 2016; Morin, Arens, \& Marsh, 2015; Reise, 2012) posibilitan diferenciar la varianza vinculada a un hipotético factor general que subyace a todos los ítems, de aquella relacionada con dimensiones que aportan información específica sobre dicho factor. La estimación se realizó por medio de "explorating structural equation modeling" (ESEM). Tres razones fundamentaron esta elección. Primero, ESEM proporciona una representación mucho mejor de los datos en términos de ajuste, especialmente, para fines confirmatorios (Guay, Morin, Litalien, Valois, \& Vallerand, 2015; Marsh, Morin, Parker, $\&$ Kaur, 2014). Segundo, conserva las virtudes del Análisis Factorial Confirmatorio (AFC), pero es más flexible y tiene menos errores de identificación y especificación que éste (Guay et al., 2015; Marsh et al., 2014). Por último, logra estimaciones más exactas de las relaciones entre factores latentes (Asparouhov \& Muthén, 2009; Morin, Marsh, \& Nagengast, 2013; Schmitt \& Sass, 2011). Se utilizó el programa Mplus 8.4 
Tabla 1. Población y muestra

\begin{tabular}{lccccc}
\hline Institución & Matrícula & \% Poblacional & Muestra & $\%$ Muestral & \% Total representado \\
\hline Universidad privada 1 & 2,036 & 50.9 & 327 & 37.9 & 16.1 \\
Universidad privada 2 & 808 & 20.2 & 175 & 20.3 & 21.7 \\
Universidad pública & 1,151 & 28.8 & 358 & 41.6 & 31.1 \\
\hline
\end{tabular}

Tabla 2. Escala de Prosocialidad de Caprara, Steca, Zelli y Capanna (2005)

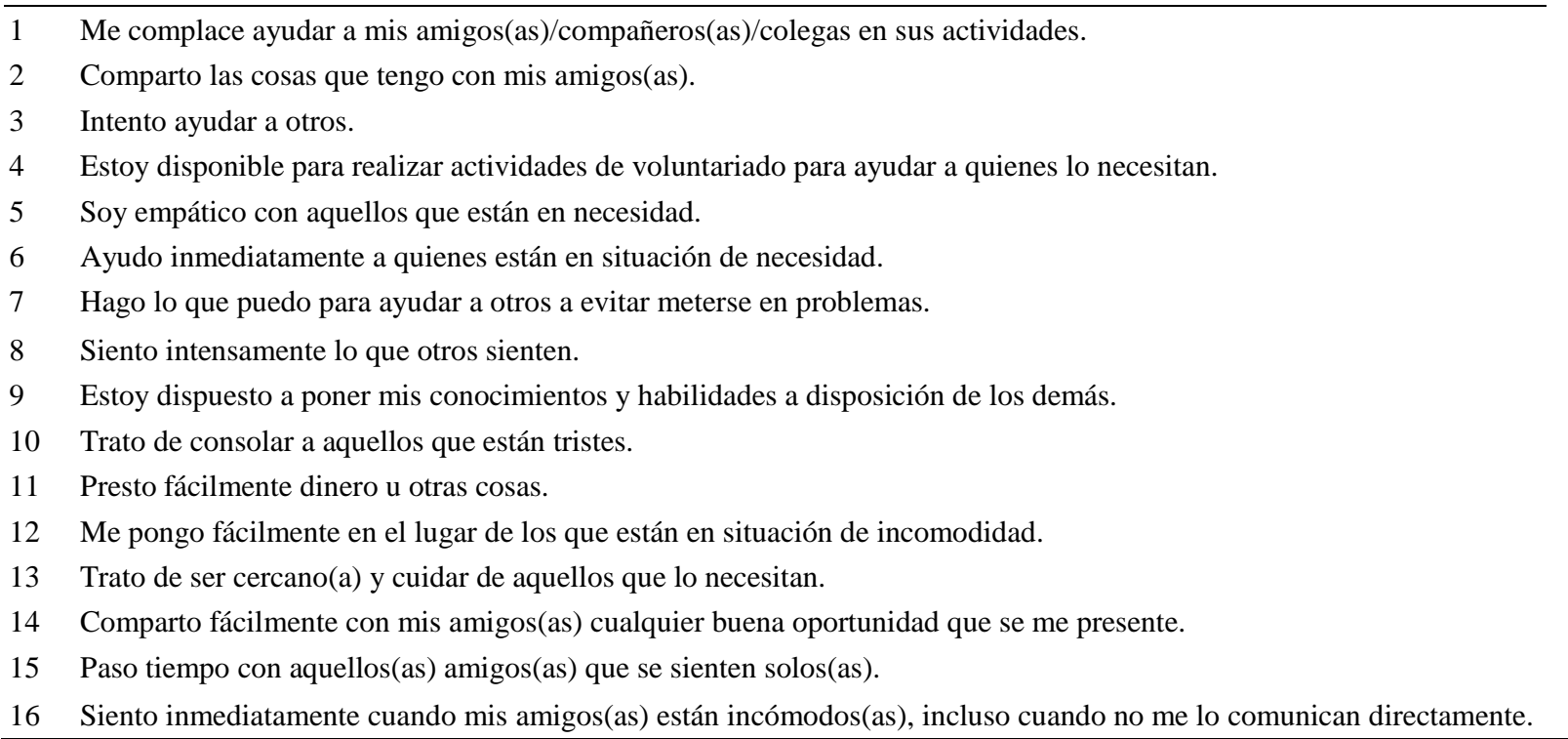

(Muthén \& Muthén, Copy 1998-2019). La Figura 1 muestra la representación conceptual del modelo.

Para la estimación del modelo se utilizó rotación target, que permite usar ESEM en modo confirmatorio, dado que produce la solución rotada más cercana a una configuración preespecificada de cargas (Asparouhov \& Muthén, 2009). Ello proporciona un modelo a priori más robusto y facilita la interpretación de los resultados (Marsh et al., 2014).

Los indicadores utilizados para evaluar la unidimensionalidad de la escala fueron Explained Common Variance (ECV), Percent of Uncontaminated Correlations (PUC) y Percentage of Reliable Variance (PRV) (Bonifay, Reise, Scheines, \& Meijer, 2015; Brunner, Nagy, \& Wilhelm, 2012; S. Reise, 2012; Rios \& Wells, 2014). Valores de ECV >.70 y PUC > .70 indican que el sesgo relativo será leve y la variación común puede considerarse esencialmente unidimensional (Rodriguez, Reise, \& Haviland, 2016). Si el PUC es mayor a .80, los valores de ECV son menos importantes prediciendo el sesgo (Reise, Scheines, Widaman, \& Haviland, 2013). Con relación al PRV, valores superiores a .75 indican fuerte evidencia en favor de usar score de alguna subescala (Li, Toland, \& Usher, 2016).

Se estimó la fiabilidad con los índices Omega de McDonald $(\omega)$, Omega relativo ( $\omega \mathrm{s})$, Omega jerárquico $(\omega \mathrm{h})$ (Reise et al., 2013; Rodriguez et al., 2016; Zinbarg, Revelle, Yovel, \& Li, 2005) y el Coeficiente H (Hancock \& Mueller, 2001). Particularmente, el $\omega \mathrm{h}$ estima de manera más precisa la fuerza de un factor general en modelos de ecuaciones estructurales (Gignac, 2014, 2015), además de permitir un claro contraste con el peso de los factores específicos (Brunner et al., 2012). Valores mayores a .75 indican predominio de un único factor general (Reise et al., 2013). Respecto del Coeficiente $\mathrm{H}$, valores superiores a .80 sugieren una variable latente bien definida (Rodriguez et al., 2016).

El modelo se estimó mediante mínimos cuadrados no ponderados con ajuste en medias y varianzas, WLSMV. Éste utiliza la matriz de correlaciones policóricas que toma en consideración la naturaleza ordinal de los ítems. La bondad de ajuste se evaluó a través de los siguientes indicadores: índice de ajuste comparativo (CFI), Tucker-Lewis Index (TLI) y Error cuadrático medio de aproximación (RMSEA). 


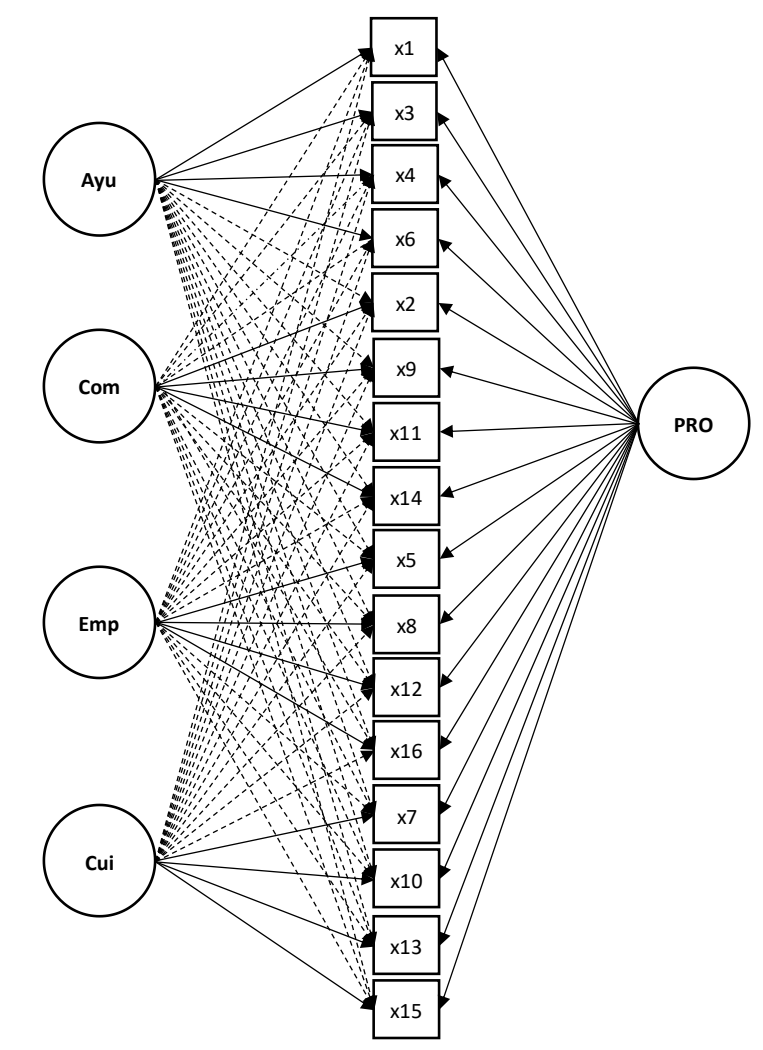

Figura 1. Modelo ESEM bifactor de la Escala de

Prosocialidad de Caprara et al., (2005).

$\mathrm{PRO}=$ Prosocialidad; $\mathrm{Ayu}=$ Ayudar;

Com=Compartir; Emp=Sentir Empatía;

Cui=Cuidar.

Para fines interpretativos, se considera un ajuste adecuado, si el CFI y TLI tienen valores superiores a .90 (Schumacher \& Lomax, 1996). Mientras que para el RMSEA son razonables valores inferiores a .08 (Browne \& Cudeck, 1993).

\section{Resultados}

Para el modelo evaluado, los índices de bondad de ajuste desplegaron los siguientes valores: $\mathrm{CFI}=.993$; TLI=.982; RMSEA $=.035 \mathrm{e}$ IC $90 \%=(.025$.044 $)$. Estos corroboran que el modelo presenta un ajuste adecuado. La Tabla 3 muestra las cargas factoriales estandarizadas, como todos los demás índices calculados.

Las cargas en el factor general de prosocialidad presentaron un rango que varió entre .75 y .33 , todas significativas. Las cargas de los factores específicos en la dimensión primaria, resultaron más bajas, siete de ellas fueron estadísticamente significativas, pero con pesos mayoritariamente inferiores a .3 y una presentó un valor negativo. Excepcionalmente, la dimensión referida a sentimiento empático tuvo valores relativamente más elevados, con el $75 \%$ de sus ítems por sobre .30. Todos los indicadores para evaluar unidimensionalidad de la escala superaron sus puntales de corte (ver Tabla 3). El valor más alto de ECV se obtuvo para el factor general y fue de .76. El valor de PUC para esta escala fue de .80 , superior al puntaje de corte propuesto de .80 , indicando que el valor de ECV es menos importante prediciendo el sesgo y es un buen indicador para pensar en la unidimensionalidad del test (Reise et al., 2013). Asimismo, el $\omega$ h del factor general fue de .864. Por lo tanto, PUC $>.80$, ECV $>.60$ y $\omega \mathrm{h}>.70$ indican que el test es esencialmente unidimensional (Reise et al., 2013). En cambio, los factores específicos desplegaron valores por debajo de los puntos de corte. Todos los valores de Omega para el factor general superaron el valor de .85. El PRV fue de 90.4\%, lo que indica fuerte evidencia en favor de usar el score total. Todos los valores de PRV para las subescalas estuvieron por debajo de .50 , lo que indica fuerte evidencia contradiciendo el uso de los scores de alguna de ellas.

\section{Discusión}

El objetivo del estudio fue evaluar la consistencia interna, fiabilidad, estructura factorial y validez convergente de la Escala de Prosocialidad Adulta de Caprara et al. (2005) con estudiantes de pedagogía chilenos. En virtud de ello, se analizó la estructura interna del instrumento por medio de un modelo ESEM bifactorial. El modelo propuesto mostró un buen ajuste y presentó propiedades psicométricas adecuadas para medir prosocialidad en personas que, según su edad, pueden ser consideradas adolescentes tardíos o adultos emergentes (Barrera \& Vinet, 2017; UNICEF, 2011). Estos resultados coinciden con los de otras investigaciones -además de la original- que corroboran la validez y fiabilidad general del instrumento para medir prosocialidad en personas de rango etario similar (Caprara et al., 2012; Caprara \& Steca, 2007; Capurso et al., 2016; Luengo et al., 2014; Suriá, 2017). Asimismo, en una realidad cultural distinta de la original, como 
Tabla 3. Cargas factoriales estandarizadas resultantes del modelo bifactor B-ESEM e indicadores de unidimensionalidad y fiabilidad de la escala de Caprara et al (2005)

\begin{tabular}{|c|c|c|c|c|c|c|}
\hline Ítems & Factor teórico & $\begin{array}{l}\text { Factor } \\
\text { general }\end{array}$ & Factor 1 & Factor 2 & Factor 3 & Factor 4 \\
\hline 1 & Ayu & $.70(.04)^{*}$ & $.49(.10)^{*}$ & $.09(.03)$ & $-.12(.05)^{*}$ & $-.07(.03)$ \\
\hline 3 & Ayu & $.74(.02)^{*}$ & $.12(.08)$ & $-.02(.04)$ & $-.14(.04)^{*}$ & $-.07(.04)$ \\
\hline 4 & Ayu & $.58(.03)^{*}$ & $-.16(.08)$ & $-.05(.04)$ & $-.18(.04)^{*}$ & $-.05(.04)$ \\
\hline 6 & Ayu & $.69(.03)^{*}$ & $-.35(.09)^{*}$ & $-.02(.03)$ & $-.11(.05)$ & $-.06(.04)$ \\
\hline 2 & Com & $.53(.03)^{*}$ & $.13(.05)^{*}$ & $.40(.08)^{*}$ & $.04(.04)$ & $-.18(.05)^{*}$ \\
\hline 9 & Com & $.56(.03)^{*}$ & $.11(.04)^{*}$ & .07 (.05) & $-.01(.04)$ & $.08(.05)$ \\
\hline 11 & Com & $.33(.03)^{*}$ & $.14(.05)^{*}$ & $.26(.07)^{*}$ & $.20(.05)^{*}$ & $-.09(.06)$ \\
\hline 14 & Com & $.40(.04)^{*}$ & $-.08(.04)$ & $.51(.09)^{*}$ & $-.12(.05)$ & $.40(.06)^{*}$ \\
\hline 5 & Emp & $.70(.03)^{*}$ & $-.21(.05)^{*}$ & $-.16(.04)^{*}$ & $.07(.06)$ & $-.10(.05)$ \\
\hline 8 & Emp & $.51(.03)^{*}$ & $.12(.04)^{*}$ & $-.14(.04)^{*}$ & $.40(.05)^{*}$ & $.10(.05)$ \\
\hline 12 & Emp & $.57(.03)^{*}$ & $-.03(.03)$ & $-.04(.04)$ & $.51(.05)^{*}$ & $.04(.03)$ \\
\hline 16 & Emp & $.36(.04)^{*}$ & $-.08(.05)$ & $.31(.05)^{*}$ & $.32(.07)^{*}$ & $.26(.06)^{*}$ \\
\hline 7 & Cui & $.56(.03)^{*}$ & $-.01(.04)$ & $-.02(.04)$ & $.09(.04)$ & $.21(.05)^{*}$ \\
\hline 10 & Cui & $.55(.03)^{*}$ & $.09(.04)$ & $.04(.04)$ & $.29(.04)^{*}$ & $.17(.05)^{*}$ \\
\hline 13 & Cui & $.74(.02)^{*}$ & $-.07(.03)$ & $-.03(.04)$ & $.14(.03)^{*}$ & $.26(.05)^{*}$ \\
\hline 15 & Cui & $.47(.03)^{*}$ & $.01(.03)$ & $.26(.05)^{*}$ & $.07(.04)$ & $.46(.06)^{*}$ \\
\hline \multicolumn{2}{|c|}{ Varianza común explicada (ECV) } & .760 & .058 & .071 & .060 & .051 \\
\hline \multicolumn{2}{|c|}{ Omega de McDonald: $\omega$} & .905 & .808 & .647 & .701 & .745 \\
\hline \multicolumn{2}{|c|}{ Omega jerárquico: $\omega \mathrm{h}$} & .864 & .001 & .206 & .121 & .136 \\
\hline \multicolumn{2}{|c|}{ Omega relativo: $\omega \mathrm{s}$} & .956 & .001 & .318 & .173 & .183 \\
\hline \multicolumn{2}{|c|}{ Replicabilidad de constructo: $\mathrm{H}$} & .901 & .332 & .385 & .351 & .295 \\
\hline \multicolumn{2}{|c|}{$\begin{array}{l}\text { Porcentaje de correlaciones no } \\
\text { contaminadas (PUC) }\end{array}$} & .800 & & & & \\
\hline \multicolumn{2}{|c|}{ Porcentaje de varianza confiable (PRV) } & 90.4 & 0.1 & 31.8 & 17.3 & 18.3 \\
\hline
\end{tabular}

Nota. B-ESEM=Análisis bifactor exploratorio de modelo de ecuaciones estructurales; Ayu=Ayuda; Com=Compromiso; Emp=Empatía; Cui=Cuidado por el otro; ECV=Varianza Común Esperada; ECV/i=Razón entre ECV y el número de ítems por factor; $\omega \mathrm{h}=$ Omega jerárquico. Valores remarcados en negrita indican cargas factoriales en la dimensión primaria; Valores entre paréntesis corresponden al error estándar. $*=p<.01$

es el ámbito latinoamericano (Luengo et al., 2017; Rodríguez et al., 2017; Rodríguez \& Moreno, 2016).

Los datos proporcionados por ítems que en su conjunto mostraron componentes multidimensionales de la prosocialidad resultaron consistentes con una estructura bifactorial. Por lo tanto, justificaron la decisión de indexar el grado de unidimensionalidad a través de todos los índices calculados, que evidenciaron un grado fuertemente unidimensional de la escala ( $\mathrm{Li}$ et al., 2016; Reise, 2012; Rodriguez et al., 2016). El factor general fue estable y explicó la mayoría de la varianza fiable de los ítems. El $\omega$ h del factor general reafirmó esta conclusión. Asimismo, los bajos valores $\omega \mathrm{h}$ de las dimensiones específicas sintonizan com lo expresado por la literatura respecto de estos casos. Es decir, de que existe una construcción general que explica gran parte de la varianza común en las medidas (Brunner et al., 2012). Este marcado carácter unidimensional de la escala coincidió con lo evidenciado por el estudio original realizado en Italia (2005). Asimismo, concuerda con el diseño y prueba de otro instrumento aplicado a adolescentes argentinos que incluyó 30 ítems con siete dimensiones operacionales de prosocialidad y que, igualmente, se comportó unidimensionalmente (Balabanian \& Lemos, 2018). Esto último sugiere la existencia de una fuerte estructura general de la prosocialidad más allá de sus facetas específicas. Lo débilmente reflejadas que quedaron estas últimas en el presente estudio coincidió también con la investigación original. Entre éstas, las 
dimensiones mejor situadas en términos relativos fueron las de compartir y sentir empatía. Resulta relevante la presencia de la dimensión empática, confirmada también en otros estudios como faceta particular de la prosocialidad (Auné et al., 2016; Martorell et al., 1995, 2011). O, bien, asociada a otra dimensión del constructo, como la de confortar (Auné y Attorresi, 2017). Ello, en razón de que varios autores no la consideran como componente del comportamiento prosocial.

Un aporte relevante del presente estudio fue la validación del instrumento con participantes pertenecientes a un contexto institucional particular, en este caso, la educación universitaria, específicamente, de formación inicial docente. Algo similar se había realizado en Italia, pero en el ámbito de la salud (Biagioli et al., 2016). Ello, puede ser importante en el contexto estudiado, dadas las orientaciones de la política educativa tendientes a fortalecer la convivencia escolar (MINEDUC, 2015). E, igualmente, las directrices que se orientan al fortalecimiento de los vínculos sociales y la democracia (MINEDUC, 2010), considerando los beneficios que ello entraña (Caprara et al., 2012; Carlo et al., 2010).

En distintos grados y formas de operacionalización, las acciones de ayudar, compartir, cuidar y sentir empatía, constituyen componentes de la prosocialidad en estudios realizados en contextos educativos. En algunos casos, como variable positivamente asociada con el aprendizaje y el mejoramiento de la conducta en estudiantes preadolescentes y adolescentes (Caprara et al., 2014; Caprara et al., 2015; Inglés et al., 2011). Igualmente, la optimización del trabajo docente cuando los profesores comparten recursos (Ertesvåg, 2014). Por otra parte, al incorporar el sentimiento de empatía entre sus ítems, el instrumento considera un rasgo que resulta relevado en estudios educativos. Particularmente, como característica de los maestros para promover la convivencia y el aprendizaje al interior de la escuela (Cappella et al., 2013; Denisenkova \& Nisskaya, 2016; Riley, 2010; Shin \& Ryan, 2017). Lo que impacta positivamente en el equilibrio emocional de los estudiantes (Sointu et al., 2017). Y, consecuentemente, en sintonía con las necesidades de educación social (MINEDUC, 2010, 2015; UNESCO, 1996) y emocional (Cefai et al., 2018) recomendadas por informes nacionales $\mathrm{e}$ internacionales.

Dentro de las líneas de investigación que conviene implementar a futuro, se aprecia que una segunda etapa en la evaluación de la prosocialidad en formación inicial docente exigiría la adecuación de los comportamientos genéricos y personales relevados en la presente investigación, pero adecuados al contexto educativo. Esto, considerando la variabilidad que puede presentar la prosocialidad según la situación (González, 2000) y en razón de lo sugerido por algunos autores de generar instrumentos que midan la prosocialidad en ámbitos institucionales específicos (Auné et al., 2014). A lo anterior, se añade el hecho de que la prosocialidad se modifica con la edad (Contreras y Reyes, 2009). $\mathrm{Y}$ esto, en el ámbito de la formación inicial docente resulta importante, dado que muchos de los estudiantes que ingresan a los programas de pedagogía están viviendo la transición de la adolescencia a la adultez.

Una limitación de este estudio es que todavía no se cuenta con estudios previos que midan la prosocialidad en un ámbito institucional específico de formación inicial de profesores. Ello dificulta, sin duda, la posibilidad de contrastar resultados. Luego, un desafío que emerge es la construcción de instrumentos, cuyos ítems contextualicen la prosocialidad en dicho contexto específico.

Tampoco, es posible dar cuenta de los cambios que puede experimentar la prosocialidad a lo largo de una trayectoria de formación pedagógica inicial. Más todavía, en una fase psicológica transicional de carácter crítico, como lo es el paso de la adolescencia a la vida adulta. Esto constituye una limitación por cuanto varias investigaciones han identificado la prosocialidad como un rasgo dinámico en el tiempo.

En conclusión, la escala propuesta constituye una herramienta adecuada para desarrollar estudios que busquen medir prosocialidad en estudiantes de pedagogía. Ello podría generar información que sirva de insumo para retroalimentar y potenciar los procesos formativos que demandan el desarrollo de este atributo de la profesión.

Finalmente, es recomendable que la investigación futura desarrolle estudios 
longitudinales que evalúen la evolución de las disposiciones y comportamientos prosociales en contextos de formación docente. Se trataría de observar si éstos se modifican en el tiempo y cuáles serían, eventualmente, los factores que determinarían estos cambios. Especialmente, evaluar si la prosocialidad en estos contextos puede verse afectada por la acción de los programas formativos.

\section{Referencias}

Asparouhov, T., \& Muthén, B. O. (2009). Exploratory structural equation modeling. Structural Equation Modeling, 16, 397-438.

Auné, S., Abal, F., \& Attorresi, H. (2016). Diseño y Construcción de una Escala de Conducta Prosocial para Adultos. Revista Iberoamericana de Diagnóstico y Evaluación - e Avaliação Psicológica, 2(42), 15-25.

Auné, S., \& Attorresi, H. (2017). Dimensionalidad de un Test de Conducta Prosocial. Evaluar, 17(1), 29-37.

Auné, S., Blum, D., Abal, J., Lozzia, G., \& Attorresi, H. (2014). La conducta prosocial: Estado actual de la investigación. Perspectivas En Psicología: Revista de Psicología y Ciencias Afines, 11(2), 21-33.

Balabanian, C., \& Lemos, V. (2018). Desarrollo y Estudio Psicométrico de una Escala para Evaluar Conducta Prosocial en Adolescentes. Revista Iberoamericana de Diagnóstico y Evaluación - e Avaliação Psicológica, 3(48), 177-188.

Barrera, A., \& Vinet, E. (2017). Adultez Emergente y características culturales de la etapa en universitarios chilenos. Terapia Psicológica, 35(1), 47-56.

Biagioli, V., Prandi, C., Giuliani, L., Nyatanga, B., \& Fida, R. (2016). Prosocial behaviour in palliative nurses: Psychometric evaluation of the prosociality scale. International Journal of Palliative Nursing, 22(6), 292-298.

Bonifay, W., Reise, S., Scheines, R., \& Meijer, R. (2015). When are multidimensional data unidimensional enough for structural equation modeling? An evaluation of the DETECT Multidimensionality Index. Structural Equation Modeling: A Multidisciplinary
Journal, 00, 1-13.

Browne, M., \& Cudeck, R. (1993). Alternative ways of assessing model fit. In K. Bollen \& J. Long (Eds.), Testing structural equation models (pp. 136-162). Newbury Park, CA: Sage.

Brunner, M., Nagy, G., \& Wilhelm, O. (2012). A Tutorial on Hierarchically Structured Constructs. Journal of Personality, 80(4), 796-846.

Cappella, E., Kim, H. Y., Neal, J. W., \& Jackson, D. R. (2013). Classroom peer relationships and behavioral engagement in elementary school: The role of social network equity. American Journal of Community Psychology, 52(3-4), 367-379.

Caprara, G., Alessandri, G., \& Eisenberg, N. (2012). Prosociality: The contribution of traits, values, and self-efficacy beliefs. Journal of Personality and Social Psychology, 102(6), 1289-1303.

Caprara, G., Luengo, B., Gerbino, M., Zuffiano, A., Alessandri, G., Vecchio, G., ... Bridglall, B. (2014). Positive effects of promoting prosocial behavior in early adolescence: Evidence from a school-based intervention. International Journal of Behavioral Development, 38(4), 386-396.

Caprara, G., Luengo, B., Zuffiano, A., Gerbino, M., \& Pastorelli, C. (2015). Why and how to promote adolescents' prosocial behaviors: Direct, mediated and moderated effects of the CEPIDEA School-Based Program. J Youth Adolescence, 44(12), 2211-2229.

Caprara, G., \& Steca, P. (2007). Prosocial agency: The contribution of values and self-efficacy beliefs to prosocial behavior across ages. Journal of Social and Clinical Psychology, 26(2), 218-239.

Caprara, G. V., Steca, P., Zelli, A., \& Capanna, C. (2005). A new scale for measuring adults' prosocialness. European Journal of Psychological Assessment, 21(2), 77-89.

Capurso, M., Dennis, J., \& Piobbico, G. (2016). Cultivating a better society: Anti-mafia farm participation increases prosocial motivations. International Journal of Educational Research, 75, 17-23.

Carlo, G., Mestre, M., Samper, P., Tur, A., \& Armenta, B. (2010). Feelings or cognitions? 
Moral cognitions and emotions as longitudinal predictors of prosocial and aggressive behaviors. Personality and Individual Differences, 48, 872-877.

Carlo, G., \& Randall, B. (2002). The development of a measure of prosocial behaviors for late adolescents. Journal of Youth and Adolescence, 31(1), 31-44.

Cefai, C., Bartolo, P., Cavioni, V., \& Downes, P. (2018). Strengthening social and emotional education as a core curricular area across the $E U$. A review of the international evidence, NESET II report. Luxembourg: Publications Office of the European Union.

Contreras, C., \& Reyes, I. (2009). Aproximaciones metodológicas en la medición de la conducta prosocial en niños de edad escolar. Revista Iberoamericana de Diagnóstico y Evaluación - e Avaliação Psicológica, 1(27), 29-44.

Davis, L. L. (1992). Instrument review: Getting the most from a panel of experts. Applied Nursing Research, 5, 194-197.

Denisenkova, N. S., \& Nisskaya, A. K. (2016). The role of teacher-child interaction in promoting peer communication. Psychology in Russia: State of the Art, 9(3), 173-187.

Eisenberg, N., Spinrad, T., \& Knafo-Noam, A. (2015). Prosocial Development. In M. Lamb \& R. Lerner (Eds.), Handbook of Child Psychology and Developmental science. Volume 3. Socioemotional Processes $\left(7^{\circ}\right.$, pp. 610-656). New Jersey: Wiley.

Ertesvåg, S. K. (2014). Teachers' collaborative activity in school-wide interventions. Social Psychology of Education, 17(4), 565-588.

Escobar, J., \& Cuervo, A. (2008). Validez de contenido y juicio de expertos: Una aproximación a su utilización. Avances En Medición, 6(1), 27-36.

Fisher, R. (1993). Social desirability bias and the validity of indirect questioning. Journal of Consumer Research, 20(2), 303-315.

Gignac, G. (2014). On the inappropriateness of using items to calculate total scale score reliability via coefficient alpha for multidimensional scales. European Journal of Psychological Assessment, 30, 130-139.

Gignac, G. (2015). Estimating the strength of a general factor: Coefficient omega hierarchical. Industrial and Organizational Psychology, 8(3), 434-438.

González, M. (2000). Conducta prosocial: Evaluación y intervención. Madrid: Morata.

Guay, F., Morin, J. S., Litalien, D., Valois, P., \& Vallerand, R. (2015). Application of exploratory structural equation modeling to evaluate the Academic Motivation Scale. The Journal of Experimental Education, 83(1), 5182.

Hancock, G. R., \& Mueller, R. O. (2001). Rethinking construct reliability within latent variable systems. In R. Cudeck, S. H. C. du Toit, \& D. Sörbom (Eds.), Structural Equation Modeling: Past and Present. A Festschrift in Honor of Karl G. Jöreskog (pp. 195-261). Chicago: Scientific Software International.

Howard, J., Gagné, M., Morin, A., \& Forest, J. (2016). Using bifactor exploratory structural equation modeling to test for a continuum structure of motivation. Journal of Management, $X X(\mathrm{X}), 1-27$.

Inglés, C., Martínez-González, A., Valle, A., García-Fernández, J., \& Ruiz-Esteban, C. (2011). Conducta prosocial y motivación académica en estudiantes españoles de Educación Secundaria Obligatoria. Universitas Psychologica, 10(2), 451-465.

Lawshe, C. H. (1975). A quantitative approach to content validity. Personnel Psychology, 28(4), 563-575.

Li, C. R., Toland, M. D., \& Usher, E. L. (2016). Dimensionality, scoring, and interpretation of the Short Grit Scale. Manuscript in Preparation.

Luengo, B., Eisenberg, N., Thartori, E., Pastorelli, C., Uribe, L., Gerbino, M., \& Caprara, G. (2017). Longitudinal relations among positivity, perceived positive school climate, and prosocial behavior in colombian adolescents. Child Development, OO(0), 1-15.

Luengo, B., Pastorelli, C., Zuffiano, A., Eisenberg, N., Ceravolo, R., \& Caprara, G. (2014). Trajectories of prosocial behaviors conducive to civic outcomes during the transition to adulthood: The predictive role of family dynamics. Journal of Adolescence, 37, $1529 \mathrm{e} 1539$.

Maneersriwongul, W., \& Dixon, J. (2004). 
Instrument translation process: A methods review. Journal of Advanced Nursing, 48(2), 175-186.

Marsh, H. W., Morin, A. J. S., Parker, P., \& Kaur, G. (2014). Exploratory structural equation modeling: An integration of the best features of exploratory and confirmatory factor analysis. Annual Review of Clinical Psychology, 10, 85-110.

Martorell, C., González, R., Aloy, M., \& Ferris, M. (1995). Socialización y conducta prosocial. Revista Iberoamericana de Diagnóstico y Evaluación Psicológica, 1, 73102.

Martorell, C., González, R., Ordóñez, A., \& Gómez, O. (2011). Estudio confirmatorio del Cuestionario de Conducta Prosocial (Ccp) y su relación con variables de personalidad y socialización. Revista Iberoamericana de Diagnóstico y Evaluación - e Avaliação Psicológica, 2(32), 35-52.

Ministerio de Educación, C. Fija texto refundido, coordinado y sistematizado de la Ley No20.370 con las normas no derogadas del decreto con fuerza de ley no 1, de 2005 (2010). Chile: Biblioteca del Congreso Nacional de Chile.

Ministerio de Educación, C. Fija texto refundido, coordinado y sistematizado de la Ley $\mathrm{N}^{\mathrm{o}} 20.370$ con las normas no derogadas del decreto con fuerza de ley $\mathrm{n}^{\circ} 1$, de 2005 (2010). Biblioteca del Congreso Nacional de Chile. Retrieved from https://www.leychile.cl/N?i=1014974\&f=201 $8-05-29 \& p=$

Ministerio de Educación, C. (2015). Política Nacional de Convivencia Escolar. 2015-2018. Santiago: Ministerio de Educación República de Chile.

Ministerio de Educación de Chile. (2010). Fija texto refundido, coordinado y sistematizado de la Ley No20.370 con las normas no derogadas del decreto con fuerza de ley no 1 , de 2005. Biblioteca del Congreso Nacional de Chile.

Ministerio de Educación de Chile. (2015). Política Nacional de Convivencia Escolar. 2015-2018. Santiago: Ministerio de Educación República de Chile.

Ministerio de Educación de Chile. (2017).
Estadísticas de la educación 2016. Santiago: MINEDUC.

Morin, A., Arens, A. K., \& Marsh, H. (2015). A Bifactor Exploratory Structural Equation Modeling Framework for the Identification of Distinct Sources of Construct-Relevant Psychometric Multidimensionality. Structural Equation Modeling: A Multidisciplinary Journal, 00, 1-24.

Morin, A. J. S., Marsh, H. W., \& Nagengast, B. (2013). Exploratory structural equation modeling. In G. R. Hancock \& R. O. Mueller (Eds.), Structural equation modeling: $A$ second course (pp. 395-436). Charlotte, NC: Information Age.

Muthén, L., \& Muthén, B. (2012). Mplus statistical analysis with latent variables. User's guide (7th Ed.). Los Angeles, CA: Muthén \& Muthén.

Reise, S. (2012). The rediscovery of bifactor measurement models. Multivariate Behavioral Research, 47(5), 667-696.

Reise, S. P., Scheines, R., Widaman, K. F., \& Haviland, M. G. (2013). Multidimensionality and structural coefficient bias in structural equation modeling: A bifactor perspective. Educational and Psychological Measurement, 73(1), 5-26.

Riley, P. (2010). Attachment theory and the student-teacher Relationship: A practical guide for teachers, teacher educators and school leaders. Abingdon: Routledge.

Riley, P. (2013). Attachment theory and the Student-Teacher Relationship, 4324.

Rios, J., \& Wells, C. (2014). Validity evidence based on internal structure. Psicothema, 26(1), 108-116.

Roche, R. (1982). Los orígenes de la conducta altruista en niños. Aspectos educativos y televisión en familia. Infancia y Aprendizaje, 19-20, 101-114.

Rodriguez, A., Reise, S. P., \& Haviland, M. G. (2016). Applying bifactor statistical indices in the evaluation of psychological measures. Journal of Personality Assessment, 98, 223237.

Rodríguez, L., Mesurado, B., Oñate, M., Guerra, P., \& Menghi, M. (2017). Adaptación de la Escala de Prosocialidad de Caprara en adolescentes argentinos. Revista Evaluar, 
17(2), 177-187.

Rodríguez, L., \& Moreno, J. (2016). Posturas éticas y empatía, predictores de prosocialidad y de penalización de faltas y delitos. Acción Psicológica, 13(2), 43-56.

Scheaffer, R., Mendenhall, W., \& Ott, L. (1987). Elementos de mиestreo. México: Grupo Editorial Iberoamérica.

Schmitt, T., \& Sass, D. (2011). Rotation criteria and hypothesis testing for exploratory factor analysis: Implications for factor pattern loadings and interfactor correlations. Educational and Psychological Measurement, 71(1), 95-113.

Schumacher, R., \& Lomax, R. (1996). A beginner's guide to structural equation modeling. New Jersey: Lawrence Erlbaum Associates.

Shin, H., \& Ryan, A. M. (2017). Friend influence on early adolescent disruptive behavior in the classroom: Teacher emotional support matters. Developmental Psychology, 53(1), 114-125.

Sointu, E. T., Savolainen, H., Lappalainen, K., \& Lambert, M. C. (2017). Longitudinal associations of student-teacher relationships and behavioural and emotional strengths on academic achievement. Educational Psychology, 37(4), 457-467.

Suriá, R. (2017). Relación entre conductas prosociales y participación en grupos online en jóvenes con discapacidad motora. Health and Addictions, 17(2), 57-66.

Trust, M. O. (1997). Trust introduces new translation criteria. Medical Outcomes Trust Bulletin, 5, 3-4.

UNESCO. (1996). La educación encierra un tesoro. Informe ala UNESCO de la Comisión Internacional sobre la educación para el siglo XXI, presidida por Jacques Delors. Madrid: Santillana.

UNICEF. (2011). Estado mundial de la Infancia 2011. Nueva York: Fondo de las Naciones Unidas para la Infancia.

Warneken, F., \& Tomasello, M. (2009). The roots of human altruism. British Journal of Psychology, 100, 455-471.

Zinbarg, R. E., Revelle, W., Yovel, I., \& Li, W. (2005). Cronbach's $\alpha$, Revelle's $\beta$, and McDonald's $\omega \mathrm{H}$ : Their relations with each other and two alternative conceptualizations of reliability. Psychometrika, 70(1), 123-133. 